\title{
Anabases
}

ANABASES Traditions et réceptions de l'Antiquité

19 | 2014

Varia

\section{Tra diritto e utopia. Aspetti della ricezione dell'opera di Moses I. Finley (1951-1967)}

Riccardo Di Donato

\section{(2) OpenEdition}

\section{Edizione digitale}

URL: http://journals.openedition.org/anabases/4497

DOI: 10.4000/anabases.4497

ISSN: 2256-9421

\section{Editore}

E.R.A.S.M.E.

\section{Edizione cartacea}

Data di pubblicazione: 1 aprile 2014

Paginazione: 21-28

ISSN: 1774-4296

\section{Notizia bibliografica digitale}

Riccardo Di Donato, «Tra diritto e utopia. Aspetti della ricezione dell'opera di Moses I. Finley

(1951-1967) », Anabases [Online], 19 | 2014, Messo online il 01 avril 2017, consultato il 21 octobre 2019. URL : http://journals.openedition.org/anabases/4497 ; DOI : 10.4000/anabases.4497

(c) Anabases 
Anabases 19 (2014), p. 21-28

\section{Tra diritto e utopia. Aspetti della ricezione dell'opera di Moses I. Finley (1951-1967)}

RicCARDo Di Donato

in memoria di Dick Whittaker

con intatta amicizia

A.cade stranamente molto spesso che nel lavoro di storia culturale ci si debba misurare con un fenomeno inatteso. Quando si deve valutare -soprattutto a una certa distanza temporale dalla sua conclusione- l'opera e la vita di uno studioso per il quale si dispone di una definizione, sedimentata e condivisa, si deve superare una prima difficoltà. La prima definizione annulla ogni elemento che conduca in diversa direzione. Un filologo resta tale anche se, in gioventù, ha pensato di fare l'attore o il ballerino e uno storico aperto all'antropologia appare lontano da (o addirittura incompatibile con) studi minuziosi e formalistici come quelli dell'epigrafia, soprattutto nella specie giuridica, anche se questi e molto altro hanno occupato la sua giovinezza. Più in generale, la costruzione di una fisonomia scientifica si compone sempre sulla base di due distinti ordini di fattori. Si tratta di due soggettività, una interna e una esterna, la cui confluenza finisce per affermarsi come apparentemente oggettiva. Ciascuno è, innanzi tutto, quello che personalmente si ritiene e poi, e in certo più generale senso, quello che di lui pensano gli altri. Vediamo un caso a conferma dell'assunto.

L'insieme degli studi relativi alla ricezione di Moses Israel Finley e della sua opera tende a privilegiare quello che fu della sua persona intellettuale dopo la pubblicazione 
di The World of Odysseus e il trasferimento dell'autore da New York a Cambridge ${ }^{1}$. La rivisitazione in corso attraverso l'indagine minuziosa e fruttuosa condotta da Daniel P. Tompkins negli archivi americani restituisce momenti e aspetti soprattutto relativi alle prime forme di impegno politico dello studioso, che si presentano in un contesto di notevole complessità ${ }^{2}$. Le carte finleyane custodite alla University Library di Cambridge, che mi fu dato di ordinare agli inizi degli anni Novanta presso il Darwin College, non contengono sostanzialmente documenti precedenti il trasferimento in Europa $^{3}$. Evidente prodotto di una selezione volontariamente operata dall'autore, esse permettono tuttavia di illuminare, se pure parzialmente, alcune intersezioni assai importanti. Ai frutti dell'esame archivistico ed a quelli della ovvia attenta lettura dei testi editi desidero qui aggiungere quelli relativi ai contatti diretti che ho avuto con Moses Finley, in particolare quelli che posso ricavare dagli appunti presi dopo una serie di conversazioni avute a Cambridge, giusto trent'anni fa, nel settembre del 1983.

Tra i primi protagonisti della ricezione in Francia dell'opera di Finley brilla certamente il nome di Louis Gernet, autore innanzi tutto di due recensioni al primo libro di Finley, lo studio sugli Horoi, i cippi ipotecari, soprattutto presenti sul territorio dell'Attica ${ }^{4}$. L'archivio finleyano conserva, nel dossier relativo a The World of Odysseus, una lettera assai calorosa, scritta da Gernet da Parigi il 17 gennaio del 19565:

«Cher confrère,

je suis bien en retard pour vous répondre et j'aurais bien d'excuses à vous faire. Je tiens du moins à vous dire combien je vous remercie de vos voux de nouvel an: c'est bien sincèrement que je vous adresse les miens. Lévy-Bruhl me dit qu'on pourrait vous voir dans le courant de cette année: j'en serais bien content.

Inutile de dire avec quel intérêt $j$ 'ai lu votre article de la RIDA (je note, en marge, que je ne vois plus du tout les choses de la même façon qu'en 1917, cf. p.180, n.406). Dans

1 In Francia resta fondamentale il saggio di P. VIDAL-NAQUET, «Économie et Société dans la Grèce ancienne: l'œuvre de Moses Finley", in Archives Européennes de sociologie VI (1965), p. 111-148 ristampato ne La démocratie grecque vue d'ailleurs, Paris, 1990, p. 55-119.

2 Il primo a me noto è: D.P. TompKins, "The world of Moses Finkelstein: The year 1939 in M.I. Finley's development as a historian», in M. MeCKelR (ed.), Classical Antiquity and the Politics of America. From George Washington to George W. Bush, Waco, Texas, 2006, p. 95-125; «Weber, Polanyi and Finley», History and Theory 47 (2008), p. 123-136.

3 R. Di Donato, "Dalle carte di Moses I. Finley», Opus VI-VIII (1987-1989), p. 259-321.

4 L. Gernet, Iura. Rivista internazionale di diritto romano e antico IV (1952), p. 361-367 = Les Grecs sans miracle, Paris, 1983, p. 121-129; "Horoi hypothécaires", in Studi in onore di U.E. Paoli, Firenze 1955, p. 345-353 = Anthropologie de la Grèce antique, Paris, 1968, p. 360-370.

5 MIF Papers, A30.

6 M.I. FInLEY, «Marriage, Sale and Gift in the Homeric World», Revue internationale des droits de l'Antiquité, 3e série II (1955), p. 167-194 = Economy and Society in Ancient Greece, New York, 1983, p. 233-245. 
l'ensemble, je me sens tout à fait d'accord avec vous: j’apprécie en particulier votre conclusion et les termes dans lesquels les dernières lignes posent le problème.

Je me permets de joindre à l'envoi de deux choses récentes celui d'un article plus ancien que je ne crois pas vous avoir donné et qui touche à des questions qui vous ont intéressé également.

L'Année Sociologique est toujours assez en retard: mais elle pense à votre Monde d'Ulysse dont elle ne tardera pas à parler (d'ailleurs, en bien) ${ }^{7}$.

Croyez, je vous prie, à mon bien amical souvenir.»

Il tono amichevole del messaggio dell'anziano grecista (maggiore di Finley di quasi trent'anni), dopo l'apostrofe anacronistica e solenne, si spiega con una conoscenza diretta realizzata all'interno della piccola comunità della Societé d'Histoire des Droits de l'Antiquité, una cui Session internationale si era tenuta a Nancy nel 1954. Finley ricordava ancora, nel 1983, riferendola a quel suo viaggio in Francia, una lunga, amichevole passeggiata con Louis Gernet al Jardin du Luxembourg, senza dirsi una parola, ignorando ciascuno dei due la versione orale della lingua dell'altro ${ }^{8}$.

La prima citazione gernetiana di un lavoro di Finley è del 1951 e si riferisce ad un intervento critico ${ }^{9}$ sul volume di Fritz Pringsheim, The Greek Law of Sale ${ }^{10}$. Nella lettera qui sopra pubblicata il riferimento gernetiano è al seminale articolo del 1955 , sviluppo specialistico sul terreno giuridico e (come Gernet avrebbe detto) pre-giuridico, di alcuni aspetti del suo libro omeristico. L'accenno a Henri Lévy -Bruhl, che dirigeva l'Institut de droit romain, e a una ulteriore presenza di Finley a Parigi trova conferma nella conferenza che lo studioso americano tenne all'Institut di place du Panthéon il 15 maggio del successivo 1959 su La condition des non-libres dans la Grèce ancienne, di cui è conservato, nell'Archivio dell'Institut, il testo dattiloscritto ${ }^{11}$. Si tratta, con ogni probabilità, dell'occasione del primo contatto con Jean-Pierre Vernant che avviene in un contesto molto particolare, presente Gernet, nella rarefatta atmosfera del circolo dei giusromanisti parigini cui lo stesso Vernant fu ammesso, da autonomo protagonista, solo una decina di anni dopo, per leggervi -in sostanziale omaggio a Gernet, nel frattempo (1962) scomparso, il suo testo su Le mariage en Grèce ancienne ${ }^{12}$. Il contesto di quel primo contatto di Vernant del 1959, mediato dall'anziano suo maestro grecista, al suo ultimo anno di attività prima che il male lo costringesse in casa fino alla morte,

7 L. Gernet, L'Année Sociologique III ${ }^{e}$ Série (1953-1954), Paris, 1956, p. 314-320 = Les Grecs sans miracle, Paris, 1983, p. 138-141.

8 Conversazione a Cambridge, 14 settembre 1983.

9 Seminar IX (1951), p. 72-91.

10 Weimar, 1950.

11 Cf. «The Servile Statuses of Ancient Greece», Revue internationale des droits de l'Antiquité $3^{\text {e }}$ série VII (1960), p. 165-179.

12 J.-P. Vernant, «Le mariage [en Grèce ancienne]», La Parola del Passato (1973), p. 51-79 = Mythe et Société en Grèce ancienne, Paris, 1974, p. 57-81. 
appare cioè assai diverso da quello, così vivacemente ricordato da Claude Mossé, al Congresso internazionale di storia economica di Aix-en-Provence, con Moses in camicia rossa e la moglie Mary che intona il più popolare dei canti rivoluzionari italiani.

L'insieme vale -io credo- a collocare nella giusta dimensione il secondo documento che qui pubblico, la lettera, spedita da Parigi il 3 giugno del 1966, con cui Vernant cercò di corrispondere alla richiesta finleyana di una prelettura del testo che lo studioso americano aveva composto in onore di Herbert Marcuse, il filosofo marxista di cui Moses era divenuto amico, negli anni a cavallo della guerra in cui aveva lavorato nel francofortese Institut für Sozialforschung, trasferito, come è noto, negli USA ${ }^{13}$ :

«Mon cher Finley,

J'ai reçu, avec votre sympathique lettre, vos deux études que j’ai lues aussitôt avec ce même intérêt que je trouve à tous vos écrits. Merci de tout cœur de votre envoi. Je vais passer, comme vous me le demandez, Utopianism ancient and modern à Pierre VidalNaquet, pour qu'il vous donne aussi son avis. Voici le mien. Je suis, pour l'essentiel, d'accord avec votre analyse. Les questions que je me pose portent peut-être sur une pure question de vocabulaire, mais il me semble, cependant qu'elles engagent le fond du problème, c'est-à-dire la définition même de l'utopie, comme type d'œuvre et forme de pensée spécifique (je termine cette lettre à la main, faute de secrétaire. Vous m’en excuserez!).

Dans les trois paires d'antithèses que vous proposez, la première occupe une place à part. En effet les oppositions static-dynamic ou ascetic-want satisfying et egalitarian-hierachical ne s'appliquent qu'aux man made social utopians et non aux gardens of Eden. Vos couples antinomiques ne divisent donc pas le champ global de l'utopie suivant trois axes comparables. Le schéma n'est pas:

\begin{tabular}{ll} 
man made social & static/dynamic \\
\hline Eden & \multicolumn{1}{|c}{} \\
& Egalitarian \\
& Hierarchical
\end{tabular}

mais:

Eden I Man-made egalitarian

hierarchical

13 MIF Papers, A37: M.I. FINLEY, «Utopianism ancient and modern», in The Critical Spirit: Essays in Honor of Herbert Marcuse, Boston, 1967, p. 3-20. 
[2] Donc, de votre point de vue même, l'opposition garden of Eden-man made social a une autre valeur et une autre portée que les deux suivantes. Le problème est de savoir si cette opposition n'est pas si profonde qu'elle implique une véritable différence de genre: l'utopie serait alors autre chose que le garden of Eden; elle en sortirait historiquement par un processus de transformation dont il faudrait définir les traits essentiels. Je m'explique mieux. Le thème d'un état paradisiaque originel, jardin d'Eden ou Age d'or, appartient à la pensée religieuse. C'est dans le cadre de cette pensée religieuse qu'il faut le situer et le comprendre: l'idée que l'humanité a connu à l'origine du temps, dans un passé primordial, une existence toute de pureté, de félicité, de continuelle jeunesse, apporte une réponse à un ordre défini de questions: comment s'explique l'état présent de l'humanité, livrée au malheur, spécialement vouée à la mort; d'où vient qu'hommes et dieux soient désormais séparés et opposés, pourquoi les hommes vivent-ils sans que leur vie soit une vraie vie puisqu'ils connaissent maladies, vieillissement, trépas, pourquoi ont-ils part à la connaissance sans savoir vraiment, puisqu'ils ignorent la réalité ultime, que l'avenir leur est impénétrable, que leur regard ne dépasse pas les apparences, pourquoi leur faut-il à chaque moment choisir entre des biens et des maux, quand ils n'ont pas les moyens, faute de les pouvoir clairement distinguer, de se décider à bon escient. Certes la description de la vie paradisiaque, contrastant avec les malheurs présents, mentionne l'absence d'injustices «sociales»: pas de guerre, abondance de biens excluant pour chacun la nécessité du travail, etc. Mais ces traits visent surtout à illustrer l'état de pureté que [3] connaissent les hommes à l'origine (pas de place en eux pour l'éris, ou le phtonos). Les malheurs «sociaux» ne constituent qu'un des aspects - et un aspect mineur- de ce qu'on peut appeler le problème du Mal, qui trouve son expression la plus pleine dans le fait de la Mort. C'est ainsi que chez Hésiode, tous les maux particuliers, guerre, faim, fatigue du labeur... se présentent comme les enfants d'une seule et même puissance de mal, $N y x$, mère de Trépas. En un mot, s'il faut caractériser le problème auquel répond, dans la pensée religieuse, le mythe du Garden of Eden, nous dirons qu'il est d'ordre métaphysico-théologique, et non social: origine de la mort, de l'ignorance, de la limitation, de la culpabilité humaines. La preuve qu'il en est bien ainsi? J'en vois au moins deux. $1^{\circ}$ Le thème du Garden of Eden est solidaire, dans le monde judéo-chrétien, des mythes de chute et de péché originel, dans le monde grec, du mythe de la faute prométhéenne et de la création de Pandora, avec toutes ses funestes conséquences. $2^{\circ}$ Le tableau de l'Age d'or est en tous points comparable à celui des îles des Bienheureux, qui ne se situent pas, dans le "passé» des hommes, mais dans l'au delà, un au delà [4] défini comme le contraire de la vie terrestre, comme le monde invisible et caché des dieux: quelques hommes privilégiés, en raison de leur naissance, de leur justice, ou de leur élection divine, peuvent atteindre, après cette vie un état définitif de non-mort, de non-mal et faire ainsi retour au mode d'existence propre aux dieux. Cette complète symétrie entre le tableau d'un Garden of Eden et celui d'une Cité des Morts bienheureux montre que, dans le premier cas comme dans le second, nous n'avons pas affaire à une création ni à une pensée utopique au sens propre. Qui admettra qu'on puisse parler d'utopie dans la description du paradis chrétien ou musulman, dans celle de l'île Blanche ou de l'île des Bienheureux chez les Grecs? 
Pour qu'il y ait pensée utopique il faudra que le thème du Garden of Eden, sans nécessairement cesser pour autant d'être religieux, se déplace de l'au delà pour se situer dans la vie terrestre: il faudra que le temps mythique, l'âge originel, vienne s'inscrire dans le temps historique humain. À cet égard, le courant judéo-chrétien a joué un rôle décisif: par l'attente du Messie ou par l'affirmation de la survenue à tel moment du temps, il a inséré le mystère religieux dans la trame de l'histoire humaine. C'est, me semble-t-il, le messianisme et ses transpositions chrétiennes qui, en bouleversant l'image et la fonction du Garden of Eden dans la pensée religieuse, ont finalement permis à l'utopie [5] de se constituer dans ce qu'elle comporte d'original. D'autant que le Messie, attendu ou reconnu comme présent à tel moment de l'histoire, a pu signifier pour certains l'affranchissement des servitudes sociales et nationales qui pesaient sur le peuple élu, pour d'autres la fin des temps, la libération du Mal, la parousie, l'âge d'or. Par là les aspects sociaux du mal et ses aspects métaphysico-religieux se trouvaient peut-être plus directement mis en rapport, confrontés. Ce qui est sûr, en tout cas, c'est que l'état de pureté et de bonheur, autrefois connu comme appartenant au passé originel ou à l'au delà de la vie, apparaissait désormais comme devant se réaliser dans un avenir peut-être proche. La voie se trouvait par là ouverte pour une pensée et une imagination authentiquement utopistes.

Excusez-moi ce long bavardage. Vous le voyez. Mes remarques tendent seulement à accentuer encore l'opposition que vous avez vous même soulignée. Je vais vous faire parvenir, d'autre part, une étude de Gernet sur les utopies hellénistiques (il y marquait [...] les mentions de la Cité du soleil et le [6] tableau traditionnel de la Cité des morts); je ne sais si vous le connaissez. J'en ai fait faire une photocopie à votre intention ${ }^{14}$.

Toutes mes amitiés à Mme Finley et bien affectueusement à vous.

Jean-Pierre Vernant»

Si tratta, come ogni lettore autonomamente apprezzerà, di una riflessione assai impegnata e sviluppata, interamente svolta sul terreno del pensiero antico, ricondotto entro una griglia interpretativa strutturale. Il sostantivo che definisce la massima utopia dei secoli XIX e XX, il socialismo, una utopia tanto importante nella vita dello studioso francese, non è neanche menzionato.

Nella sua lunga esperienza intellettuale Jean-Pierre Vernant ha sempre tenuto ben distinte le due dimensioni del suo impegno, quella verso il passato della storia del pensiero dei Greci e quella del presente della ideologia e della pratica politica: la sua prudenza nella intersezione tra i due insiemi non trova eccezioni nella sua opera intellettuale.

Io credo che, nello scrivere il suo testo, Vernant abbia volontariamente scelto, come destinatario, il primo Finley da lui conosciuto in place du Panthéon, quello mediato da Gernet, e non quello incontrato da Claude Mossé, negli intervalli del

14 L. GERNET, «La cité future et le pays des morts», Revue des études grecques XLVI (1933), p. 293-310 = Anthropologie de la Grèce antique, Paris, 1968, p. 139-153. 
congresso di storia economica, ad Aix-en-Provence. Non può tuttavia passare in ogni caso inosservata la totale assenza di ogni vibrazione politica nella prelettura di un saggio, come quello finleyano, che appare, soprattutto oggi, scritto con franca intenzione ideologica, esplicitamente rivolta al dedicatario Marcuse e alla sua storia umana e intellettuale.

Di ciò trovo conferma - e contrario - nell'ultimo testo che qui pubblico dall'archivio finleyano e cioè la risposta di Herbert Marcuse a Moses Finley, spedita da La Jolla (California) il 22 settembre del 1967:

«Dear Moe:

You may not believe it, but your letter arrived when I was reading your essay in The Critical Spirit. It is the first contribution I read -for one reason or other. I was particularly touched by the fact that you are among the contributors. Let me retaliate: if you are happy and proud to lead off, I am happy and proud that it is you who leads off. You are one of the few scholars whom I know and whom I admire -in fact: envy. Because I am a frustrated scholar...

I like your essay very much, especially your analysis of the decisive difference between ancient and early modern, and the contemporary utopias, and your conclusion that the latter are satirized precisely because they are so realistic and realizable. Conversely, the idea of socialism, for example, loses all power to the extent to which it is purged of its alleged utopian elements. Somebody should show this is a little book entitled Die Entwicklung des Sozialismus von der Wissenschaft zur Utopie...

There is only one point where I disagree with you, namely, your classification of Fourier as belonging to the lunatic fringe. He was mad, but his madness was precisely that creative imagination which projects real possibilities (if not necessities). His idea of "sweetening the oceans" is already being translated into reality with the use of atomic energy, and his basic concept of a social division of labor according to instinctual needs (travail attrayant) appears today not only as a technical possibility but perhaps also as the qualitative difference between a genuinely socialist society and the present societies.

I only wished we could discuss these questions personally. Next year? In the meantime, let me tell you again how happy and proud I am to have you among the contributors to this beautiful and indeed undeserved volume. Many, many thanks [...]

As always

Herbert ${ }^{15}$ ".

Il rapporto tra interlocutori, incardinati in istituzioni universitarie assai lontane e immersi in situazioni politico-culturali assai diverse, non può che configurarsi sotto la specie della complessità. La ricezione del destinatario appare ovviamente diversa dal quella del collega, pur intellettualmente vicino, richiesto di una prelettura. Più libera 
certamente di cogliere e sottolineare l'aspetto del messaggio che richiamava una diversa stagione ed un rapporto di interlocuzione che, nel quadro non sempre lieto dei rapporti con i francofortesi, Moses Finley ricordava come una piacevole e solare eccezione, legata a comprensione ed amicizia ${ }^{16}$. Il vero del messaggio resta intatto, uno e trino prima di moltiplicarsi nella nostra finale ricezione.

Riccardo Di Donato

Università di Pisa

r.didonato@flcl.unipi.it

16 Conversazione a Cambridge, 23 sept. 1983. 